\title{
LncRNA GAS5 promotes apoptosis as a competing endogenous RNA for miR-21 via thrombospondin 1 in ischemic AKI
}

\author{
Xuemei Geng ${ }^{1}$, Nana Song ${ }^{1}$, Shuan Zhao ${ }^{1}$, Jiarui Xu' ${ }^{1}$, Yong Liư', Yi Fang ${ }^{1}$, Mingyu Liang ${ }^{2}$, Xialian Xu and \\ Xiaoqiang Ding ${ }^{1}$
}

\begin{abstract}
Mounting evidence has indicated that long noncoding RNAs (IncRNAs) and microRNAs (miRNAs) played important roles in renal ischemia/reperfusion (I/R) injury. However, the involvement of IncRNA growth arrest specific 5 (GAS5) in acute kidney injury (AKI) remained largely unexplored. This study aimed to determine possible mechanisms of GAS5 in the renal I/R process. We found that GAS5, noticeably upregulated by renal I/R injury, was further suppressed by delayed IPC while knockdown of miR-21 in vivo before IPC could significantly increased the GAS5 levels. Concurrently, TSP-1 was negatively regulated by miR-21 in vivo and vitro. Additionally, Reciprocal repression of GAS5 and miR-21 was identified. Knockdown of miR-21 in H6R0.5 treated HK-2 cells promoted apoptosis. Co-transfection of miR-21 mimic and pcDNA-GAS5 or pcDNA-Vector were performed, results of which showed that inhibition of miR-21 on TSP-1 could be rescued by overexpression of GAS5. This study suggested that GAS5 facilitated apoptosis by competitively sponging miR-21, which negatively regulated TSP-1 in renal I/R injury. This novel regulatory axis could act as a therapeutic target for AKI in the future.
\end{abstract}

\section{Introduction}

Acute kidney injury (AKI) is a common complication characterized by a decline in renal function. Renal ischemia/reperfusion (I/R) insult contributes greatly to AKI. However, the established underlying mechanisms of $I / R$ injury are only the tip of the iceberg. Cell apoptosis is considered to be involved in pathogenesis of I/R injury. It has been reported that many mechanisms contribute to apoptosis regulation in renal tubular epithelial cells in the process of renal $I / R^{1}$.

miR-21, an important hypoxia-responsive miRNAs, was originally found in a cancer study due to its anti-apoptotic

\footnotetext{
Correspondence: Xialian Xu (xu.xialian@zs-hospital.sh.cn) or

Xiaoqiang Ding (ding.xiaoqiang@zs-hospital.sh.cn)

'Department of Nephrology, Zhongshan Hospital, Fudan University; Shanghai Institute of Kidney and Dialysis; Shanghai Key Laboratory of Kidney and Blood Purification; Shanghai Medical Center of Kidney Disease, Shanghai, China

2Department of Physiology and Center of Systems Molecular Medicine, Medical College of Wisconsin, Milwaukee, WI, USA

Edited by R. Killick
}

function. Our work demonstrated that miR-21 contributed to renal protection by reducing apoptosis via inhibiting programmed cell death protein 4 (PDCD4) ${ }^{2}$ and phosphatase and tensin homolog deleted on chromosome ten (PTEN) ${ }^{3}$. Thrombospondin-1 (TSP-1) with anti-angiogenesis effect, as a novel target gene of miR-21 in vascular endothelial cells ${ }^{4}$, also has pro-apoptosis function reported in the renal tubular epithelial cells from the study by Thakar et al. ${ }^{5}$.

Long noncoding RNAs (lncRNAs) are a novel class of non-protein-coding RNAs whose transcripts are over $200 \mathrm{nt}$ in length. Emerging evidence has shown that lncRNAs are involved in many biological processes by regulating gene expression at epigenetic, transcriptional, and post-transcriptional levels ${ }^{6,7}$. Hypoxia-regulated lncRNAs were identified in some tumor studies ${ }^{8}$ and an effect of $I / R$ on lncRNAs expression was uncovered as well ${ }^{9}$. Ectopic expression of lncRNA GAS5 was first found in hypoxia-treated renal tubular epithelial cells in Yu's 
study ${ }^{10}$. Our previous study suggested that GAS5 might contribute to apoptosis in renal $I / R$ injury ${ }^{11}$. However, how GAS5 regulates apoptosis induced by renal $I / R$ remains unclear. In addition, the negative regulation of GAS5 by miR-21 was found in breast cancer ${ }^{12}$. Thus, in this study, we will explicit: (1) the protection of miR-21 in renal I/R injury-induced cell apoptosis might be related with inhibition of GAS5 and TSP-1, (2) GAS5 as a competing endogenous RNA (ceRNA) of miR-21 might contribute to renal I/R injury by regulating expression of TSP-1.

\section{Results}

miR-21, GAS5, and TSP-1 expression and apoptosis in mice kidneys after $\mathbf{I} / \mathbf{R}$

In our previous study, we found that lncRNA GAS5 might contribute to renal $I / R$ injury due to its proapoptosis function ${ }^{11}$. However, the mechanism of GAS5 involvement in I/R-induced apoptosis remains unknown. The expression levels of miR-21, GAS5, and TSP-1 in mice kidneys were detected at diverse reperfusion intervals $(6$, 12,24 , and $48 \mathrm{~h}$ ) after ischemia for $35 \mathrm{~min}$. As illustrated in Fig. 1a, the levels of serum creatinine were significantly higher in renal I/R groups than that in Sham group.

The time-course analysis indicated that mRNA levels of GAS5 and TSP-1 were increased after I/R and peaked at $12 \mathrm{~h}$ of reperfusion, and miR-21 expression increased gradually until $48 \mathrm{~h}$ of reperfusion (Fig. 1b-d). The protein expression of TSP-1 was almost undetectable in Sham-operated kidneys, peaked at $12 \mathrm{~h}$ and decreased to the baseline level at $48 \mathrm{~h}$ of reperfusion, which was consistent with our RT-PCR results and similar to the results from Thakar's study (Fig. 1f). miR-21 and TSP-1 in I/R $(24 \mathrm{~h})$ treated kidneys were expressed mainly in renal tubular epithelial cells, as shown in the ISH results (Fig. 1e). Therefore, we hypothesized that a relationship among miR-21, GAS5, and TSP-1 might exist.

In addition, our results revealed that the percentages of apoptotic cells in mice kidney tissues were higher in the I/ $\mathrm{R}$ groups than that in the Sham group, as well as the severity of renal injury assessed by histopathological manifestations (Fig. 1g).

The preconditioning-induced upregulation of miR-21 accompanied by downregulated expression of GAS5 and TSP-1 contributed to renal protection of delayed IPC

To further determine the pro-apoptotic function of GAS5 in ischemic AKI and the relationship among the three genes, mice were divided into two groups: an IPC $+\mathrm{I} / \mathrm{R}$ group and a Sham $+\mathrm{I} / \mathrm{R}$ group. A schematic diagram depicting the animal treatment procedure was demonstrated in Fig. 2a. Similar to our previous studies ${ }^{2}$, IPC significantly improved renal function and markedly alleviated histopathologic damage (Fig. 2b-d), as well as decreased the percentages of apoptotic tubular cells (Fig. 2e). Additionally, miR-21 levels in mice kidneys were remarkably higher for the IPC + I/R group than the Sham $+\mathrm{I} / \mathrm{R}$ group, accompanied by downregulated TSP-1 mRNA and protein expressions (Fig. 2f, g). GAS5 expression induced by $\mathrm{I} / \mathrm{R}$ insult in mice renal tissues was significantly downregulated by delayed IPC (Fig. 2f), which suggested a possible negative correlation between GAS5 and miR-21.

\section{Knockdown of miR-21 promoted apoptosis might by upregulating GAS5 and TSP-1 expressions in the delayed} IPC

miR-21 knockdown has been suggested to attenuate the renal protection conferred by IPC. As shown in Fig. $3 \mathrm{~b}-\mathrm{e}$, the serum creatinine level, renal histological injury score and percentages of apoptotic cells at $24 \mathrm{~h}$ of the second reperfusion in mice receiving LNA antimiR-21 were significantly higher than those in mice with anti-scramble treatment. Concurrently, effective inhibition of miR-21 increased the mRNA and protein expressions of targeted TSP-1 (Fig. 3f, g). In addition, Fig. 3f showed that GAS5 levels were significantly higher in the anti-miR-21+IPC+IR group than in the control group. The results suggested that the protective effect of miR-21 might be mediated not only by targeted TSP-1 but also by GAS5 attenuating tubular cell apoptosis.

\section{TSP-1, a target of miR-21, contributed to $\mathrm{H} / \mathrm{R}$-induced apoptosis in renal proximal tubular epithelial cells}

Bioinformatic analysis was employed to predict the interaction between miR-21 and the 3'-UTR of TSP-1 (Fig. 4a). As demonstrated by luciferase reporter assays, co-transfection of anti-miR-21 and the TSP-1 $3^{\prime}$ UTR reporter construct group had markedly higher luciferase activity compared with co-transfection of antiscramble group, which revealed that miR-21 might regulate the expression of TSP- 1 by interactions with the $3^{\prime}$ UTR.

Additionally, H6R0.5 insult significantly upregulated the expression of miR-21 and downregulated TSP-1 protein (Fig. 4b, c) but not TSP-1 mRNA levels in HK-2 cells (Fig. 5a). Furthermore, the effective knockdown of miR-21 in H6R0.5-treated HK-2 cells by the LNA anti-miR-21 increased the expression of TSP-1 protein and apoptotic cell percentages (Fig. $4 \mathrm{~d}-\mathrm{g}$ ). Thus, these data suggested that knockdown of miR-21 could promote apoptosis in HK-2 cells via its target TSP-1.

We further verified the regulation of TSP-1 by miR-21 in miR-21+/0 mice. As illustrated, renal injury induced by $\mathrm{I} / \mathrm{R}$ was ameliorated in miR-21+/0 mice compared with miR-21 0/0 mice, indicated by serum creatinine levels, histopathological scoring and TUNEL apoptotic cell 


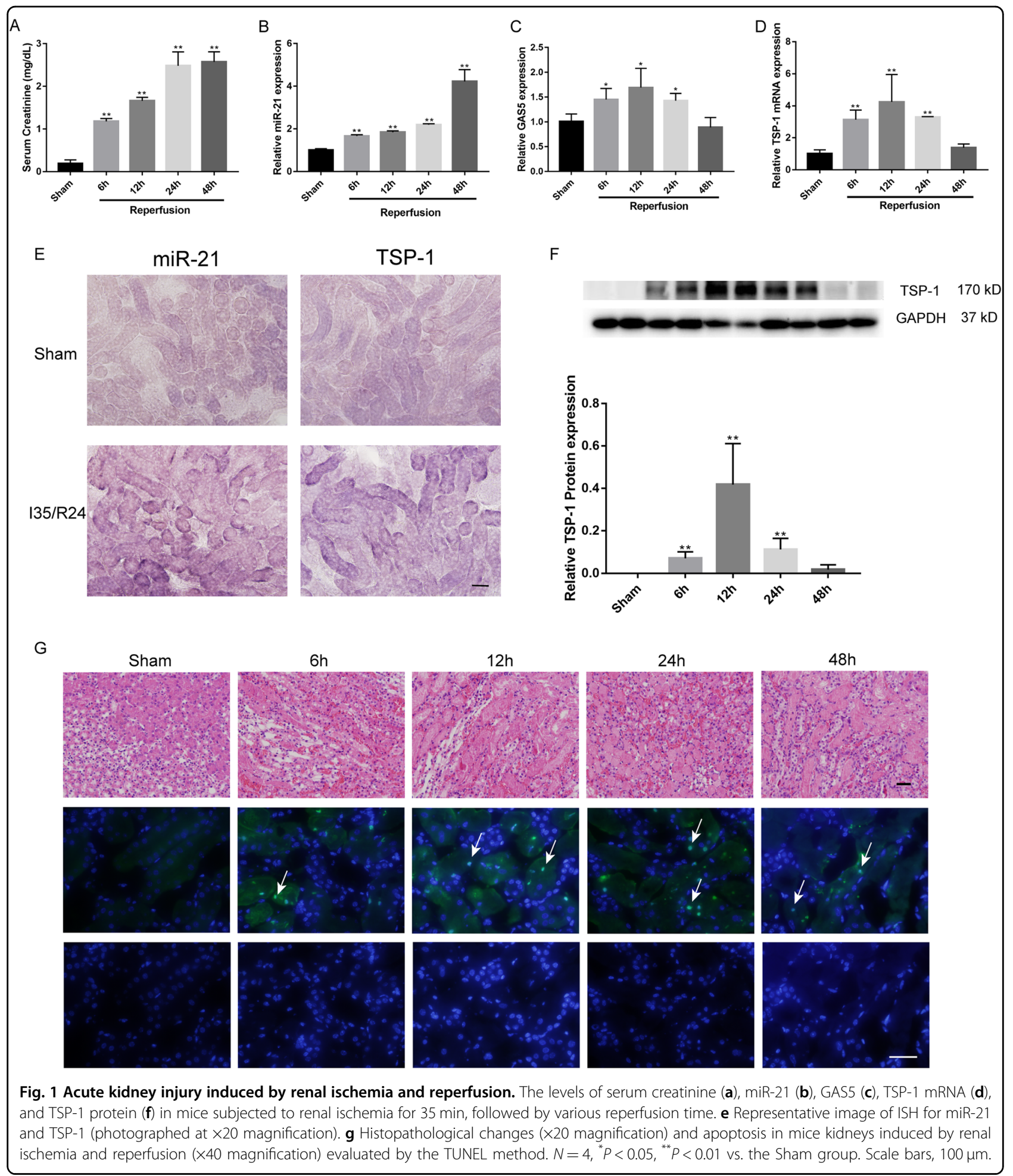

percentages (Fig. 4h-l). Concurrently, TSP-1 protein expression was downregulated upon miR-21 upregulation in miR-21+/0 mice kidneys (Fig. $4 \mathrm{~m}$ ). Thus, in vivo and in vitro, we reconfirmed the negative regulation of TSP-1 by miR-21 in renal $\mathrm{I} / \mathrm{R}$ injury.
Effects of reciprocal repression between miR-21 and GAS5 on apoptosis and TSP-1 expression in vitro

GAS5 levels were upregulated in the H6R0.5-treated cells with LNA anti-miR-21 (Fig. 5b), although H6R0.5 insult did not alter the expression of GAS5 (Fig. 5a). 


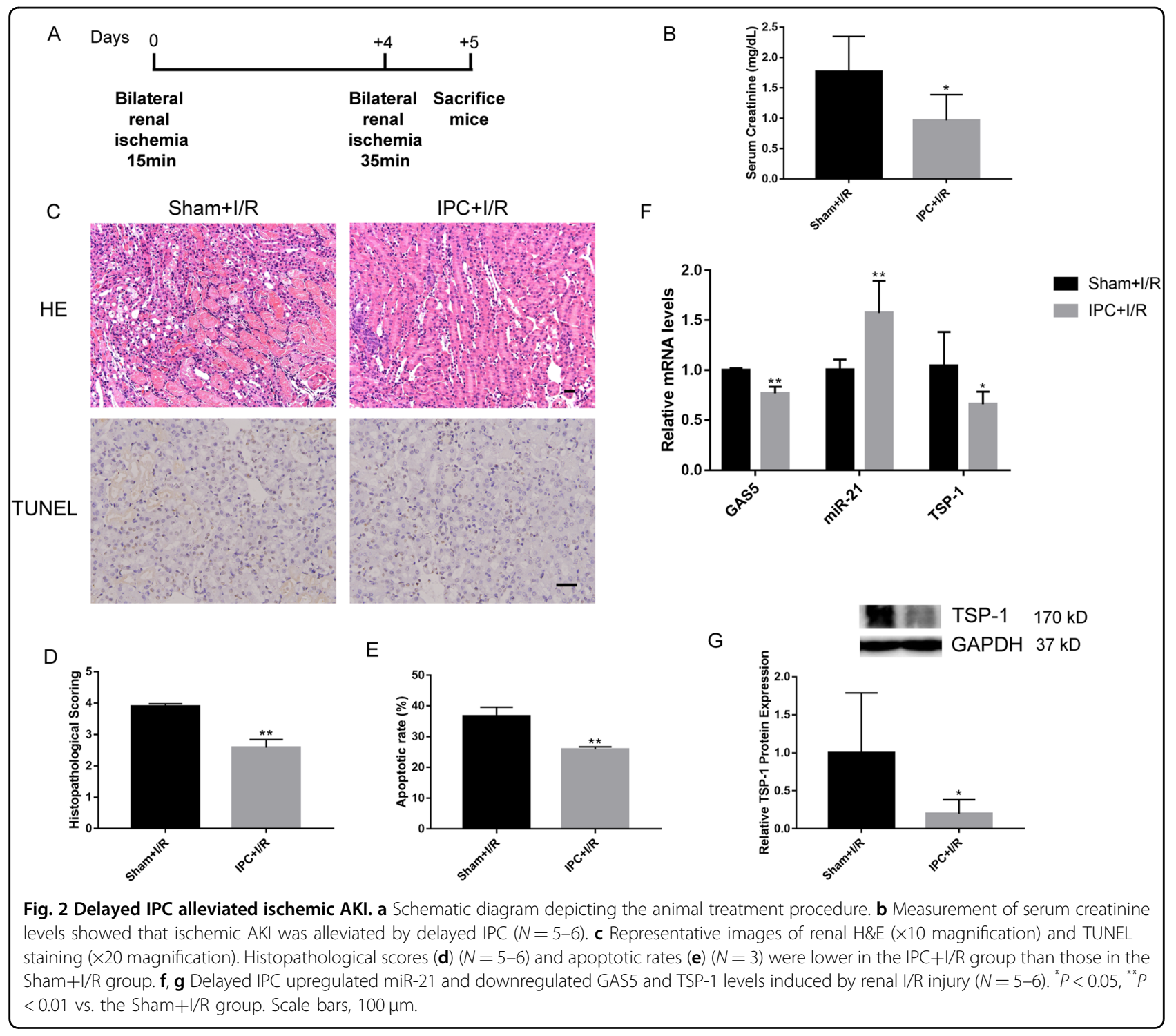

H24R3 was also established due to decreased miR-21 expression under this condition (Fig. 5c). H24R3 insult increased GAS5 and TSP-1 mRNA levels (Fig. 5c) and miR-21 mimics could suppress GAS5 and TSP-1 mRNA expression in H24R3-treated HK-2 cells, as well as TSP-1 protein abundance (Fig. $5 \mathrm{~d}, \mathrm{f}$ ). These data revealed the negative regulation of GAS 5 and TSP- 1 by miR-21 could contribute to its anti-apoptotic effects in H/R-treated HK2 cells. Interestingly, TSP-1 protein expression was downregulated in the setting of H6R0.5 and H24R3 (Figs. $4 \mathrm{c}$ and 5e), while its mRNA abundance was almost unaltered by H6R0.5 and upregulated by H24R3 insult (Fig. 5a, c).

We already clarified the role of GAS5 in cell apoptosis induced by $H / R$ insult (H24R3) in our previous work ${ }^{11}$. The RT-PCR results showed that GAS5 siRNAs resulted in miR-21 upregulation accompanied by TSP-1 downregulation in H/R-treated HK-2 cells (Fig. 5g). Conversely, overexpression of GAS5 decreased the miR-21 abundance and increased TSP-1 expression remarkably (Fig. 5h). The regulation of TSP-1 protein levels by GAS5 and its effects on apoptosis in HK-2 cells have been fully confirmed in our previous study ${ }^{11}$. Hence, the regulation of miR-21 and TSP-1 by GAS 5 could give rise to its pro-apoptotic effects in $\mathrm{HK}-2$ cells.

\section{GAS5 acted as a ceRNA for miR-21 to target TSP-1}

Zhang's study revealed that lncRNA GAS5 might contain a target site of miR-21 ${ }^{12}$. The dual-luciferase reporter assay we performed showed that miR-21 mimic, but not miR-21 nc, apparently downregulated the luciferase activity of GAS5-WT but did not alter the luciferase 


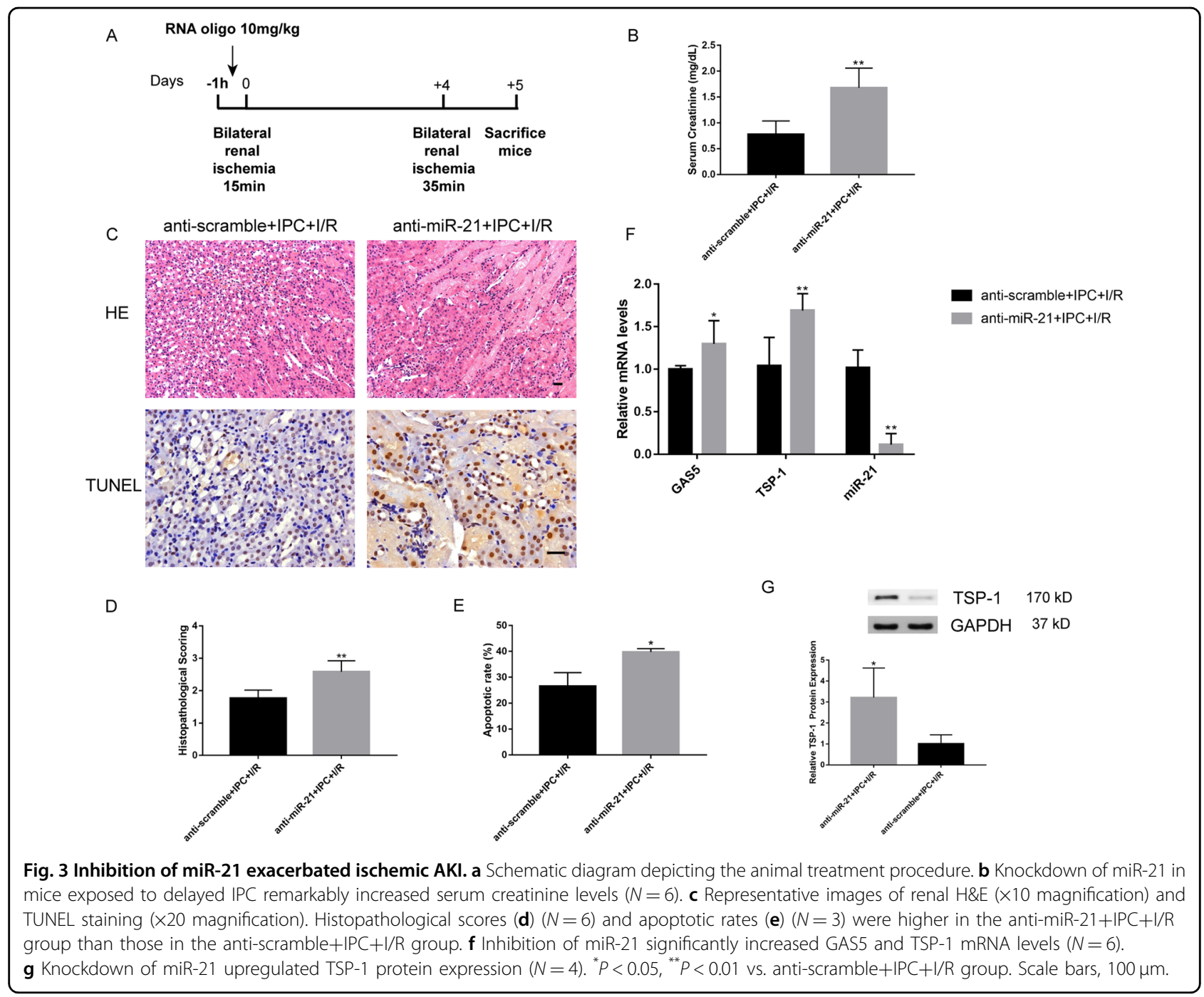

activity of GAS5-MT (Fig. 6b). Our data above suggested that GAS5 might serve as a sponge for miR-21. Furthermore, to identify the potential mechanism of GAS5 that leads to H/R-induced apoptosis, we first performed cotransfection of pcDNA-GAS5 and miR-21 mimic or nc in HK-2 cells. As shown in Fig. 6c, d, the negative regulation of TSP-1 mRNA and protein expressions by miR-21 still existed under conditions of GAS5 overexpression. Cotransfection of pcDNA-GAS5 with the miR-21 mimic could markedly decrease GAS5 abundance and cell apoptosis compared with co-transfection with miR-21 nc (Fig. 6c, e). Next, co-transfection of miR-21 mimic and pcDNA-GAS5 or pcDNA-Vector showed that inhibition of miR-21 on TSP-1 expression could be rescued and cell apoptosis was aggravated by GAS5 overexpression (Fig. $6 \mathrm{f}-\mathrm{h})$. Therefore, GAS5, as a ceRNA of miR-21, rescued the silencing effect on its target, TSP-1, and promoted apoptosis during renal I/R injury (Fig. 6i).

\section{Discussion}

LncRNA GAS5, a key regulator of cellular apoptosis, migration, and proliferation, has been widely studied in various types of tumors ${ }^{13}$. The present study indicated the relationship among GAS5, miR-21, and TSP-1 in the regulation of cell apoptosis under renal I/R condition and the underlying mechanisms.

Apoptosis is involved in the pathophysiology of ischemic AKI by complex gene regulations ${ }^{1}$. LncRNAs have been documented to have important apoptosisrelated functions in tumor studies, including GAS5 as a tumor suppressor ${ }^{14}$. GAS5 interacts with the DNAbinding domain of the glucocorticoid receptor to suppress a number of anti-apoptotic genes, such as cIAP2 ${ }^{14}$. After Lin et al. gave a landscape of lncRNAs induced by hypoxia, an increasing number of studies have explored the relationship between IncRNAs and $A K I^{15}$. GAS5 could be heavily induced in renal tissues by $I / R$ insult due 


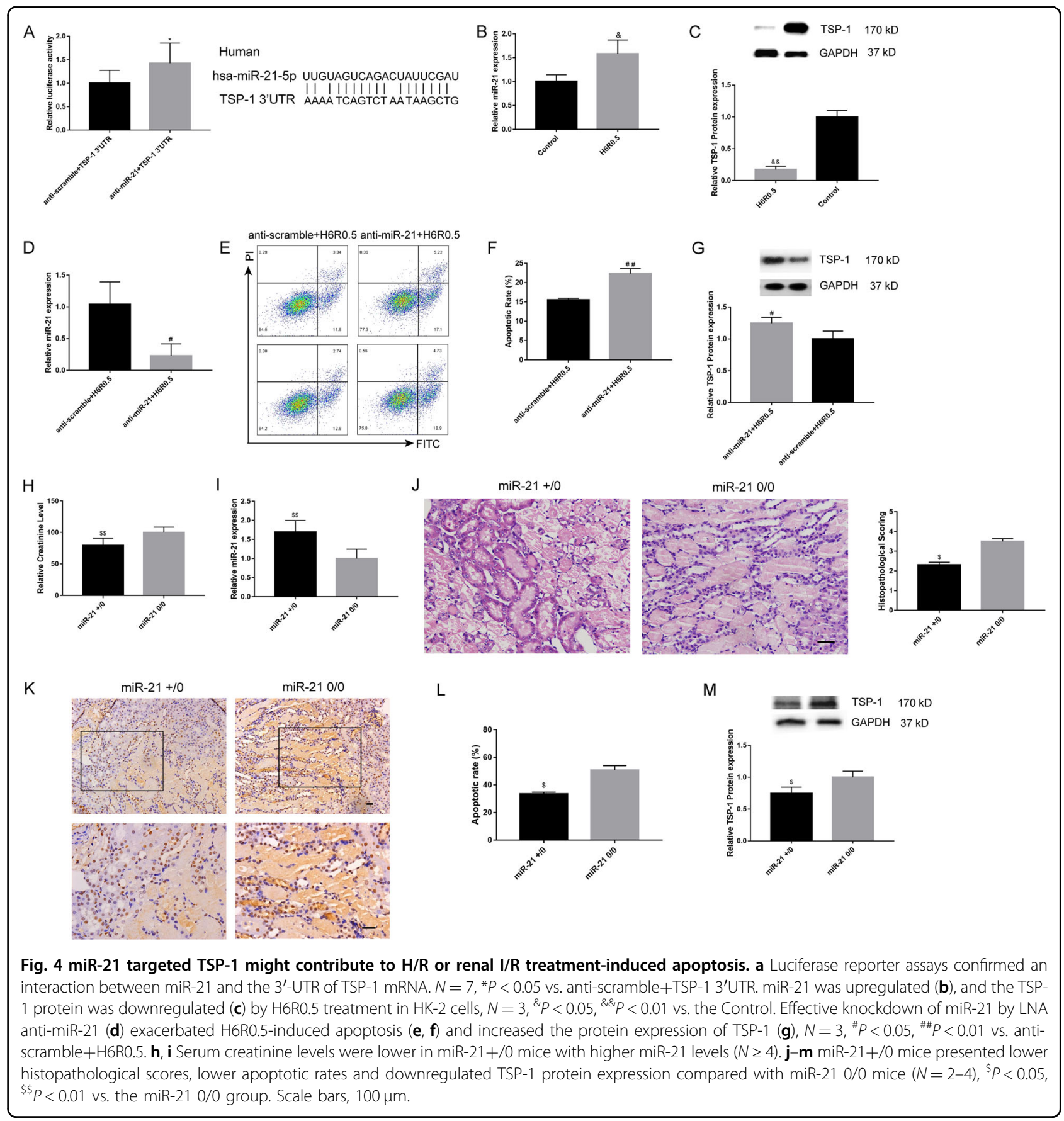

to its pro-apoptotic function ${ }^{11}$. Downregulated GAS5 might be involved in the protective effects of delayed IPC.

It was recently discovered that lncRNAs functioned as miRNA sponges. Tian's study indicated that lncRNA LINC00520 acted as a ceRNA to competitively inhibit miR$27 b-3 p$ during $I / R$ injury ${ }^{16}$. Negative regulation between miR-21 and GAS5 was first found in breast tumors ${ }^{12}$. In our study, we found negative regulation between GAS5 and miR-21 in vitro and in vivo. Moreover, luciferase reporter assays showed that GAS5 acted as a sponge to bind directly to miR-21 with a putative miRNA response element. In addition to miR-21, it has been documented that GAS5 could bind to other miRNAs as a ceRNA, including miR23a in gastric cancer ${ }^{17}$, miR-222-3p in papillary thyroid carcinoma $^{18}$, and miR-137 in ischemic stroke ${ }^{19}$.

As another type of noncoding RNA, miRNAs have been associated with the etiology and pathogenesis of AKI. Godwin et al. first compared the genome-wide miRNA 


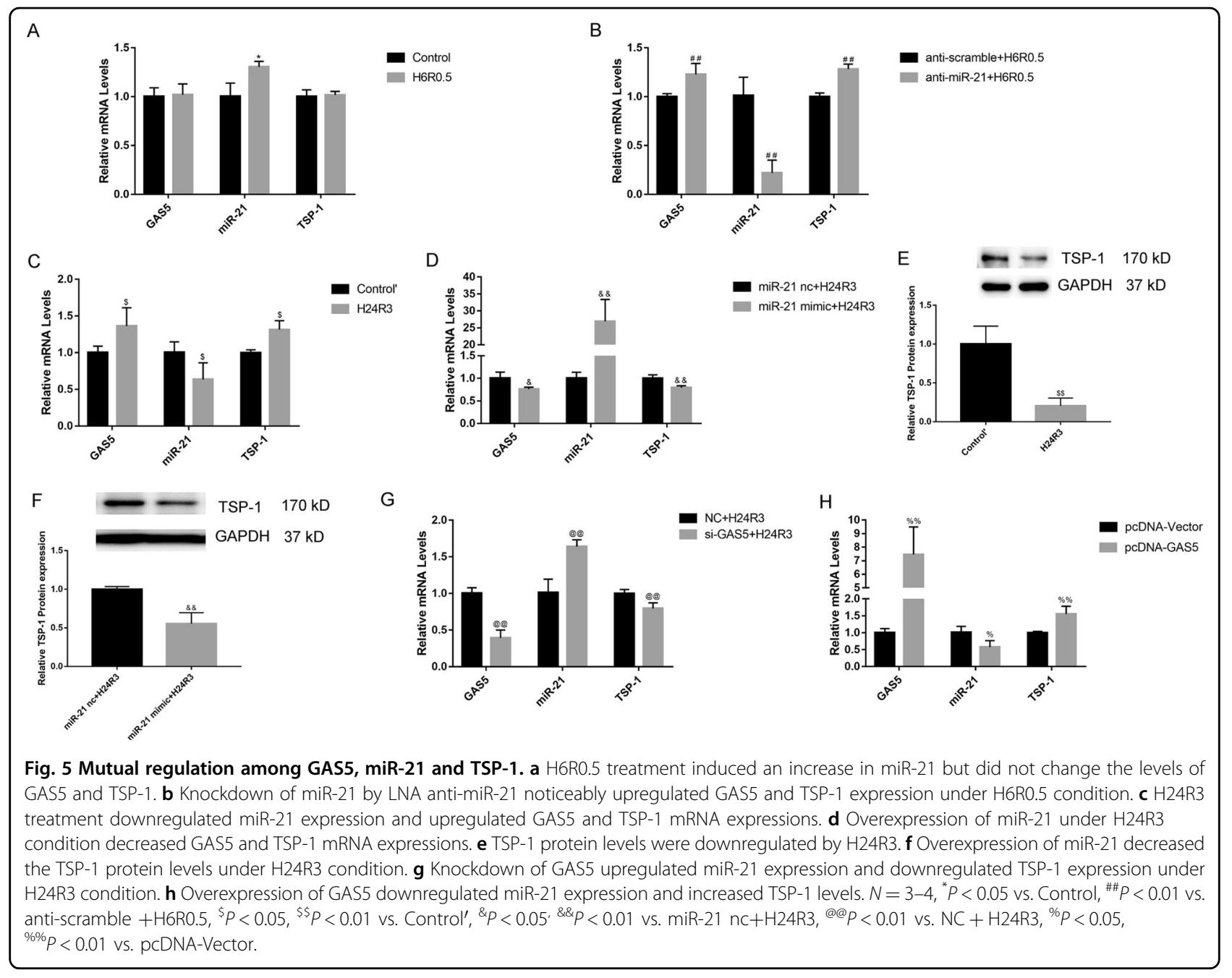

expression profile between I/R- and Sham-treated kidneys $^{20}$. Several specific miRNAs, such as miR-24, miR$30 \mathrm{a}, \mathrm{miR}-146$, and miR-210, were also investigated in subsequent studies ${ }^{21}$. Accumulating evidence has elucidated that miR-21 protected renal epithelial cells against hypoxic and inflammatory injury and participated in renal protection of delayed $\mathrm{IPC}^{2}$, xenon preconditioning ${ }^{22}$, and delayed remote $\mathrm{IPC}^{23}$ in AKI. miR-21 in urine and plasma was associated with severe AKI after cardiac surgery ${ }^{24}$. miR-21 protected kidneys mainly by targeting proapoptotic genes such as PDCD4, PTEN, and FasL ${ }^{25}$. We found and confirmed a novel target of miR-21 that regulated apoptosis during AKI in our study.

TSP-1, an important matricellular glycoprotein, has multiple functions, including anti-angiogenesis, profibrosis, pro-inflammatory, and pro-apoptosis in various cell types. TSP-1 in kidney tissues could be upregulated by ischemia $^{5}$, unilateral ureteral obstruction ${ }^{26}$, and high glucose $^{27}$, but it is rarely expressed in healthy kidneys. For the first time, Thakar et al. suggested that TSP-1 expression in renal proximal tubules was a novel regulator of I/R damage due to its pro-apoptotic function in ischemic $\mathrm{AKI}^{5}$. Additionally, the absence or disruption of TSP1-CD47 signaling protected mice from renal dysfunction and tubular damage ${ }^{28}$. Furthermore, TSP-1 could inhibit renal tubular epithelial cell proliferation and self-renewal after $I / R$ injury via activation of its receptor $\mathrm{CD} 47^{29}$. On the basis of our previous findings ${ }^{4}$, we further identified TSP-1 as a target gene of miR-21 in renal tubular epithelial cells in this study. The trend of TSP-1 expression after reperfusion in the current study was similar to the results of other studies, which was also consistent with the GAS5 expression levels. Our previous study suggested that the role of GAS5 in AKI is possibly related to TSP- $1^{11}$, and we further confirmed that TSP-1 was positively regulated by GAS5 to promote apoptosis induced by renal I/R. Crucially, TSP-1 upregulation by GAS5 could be counteracted by miR- 21 mimic, while the inhibition of TSP-1 expression by miR-21 could be rescued by GAS5 overexpression. 


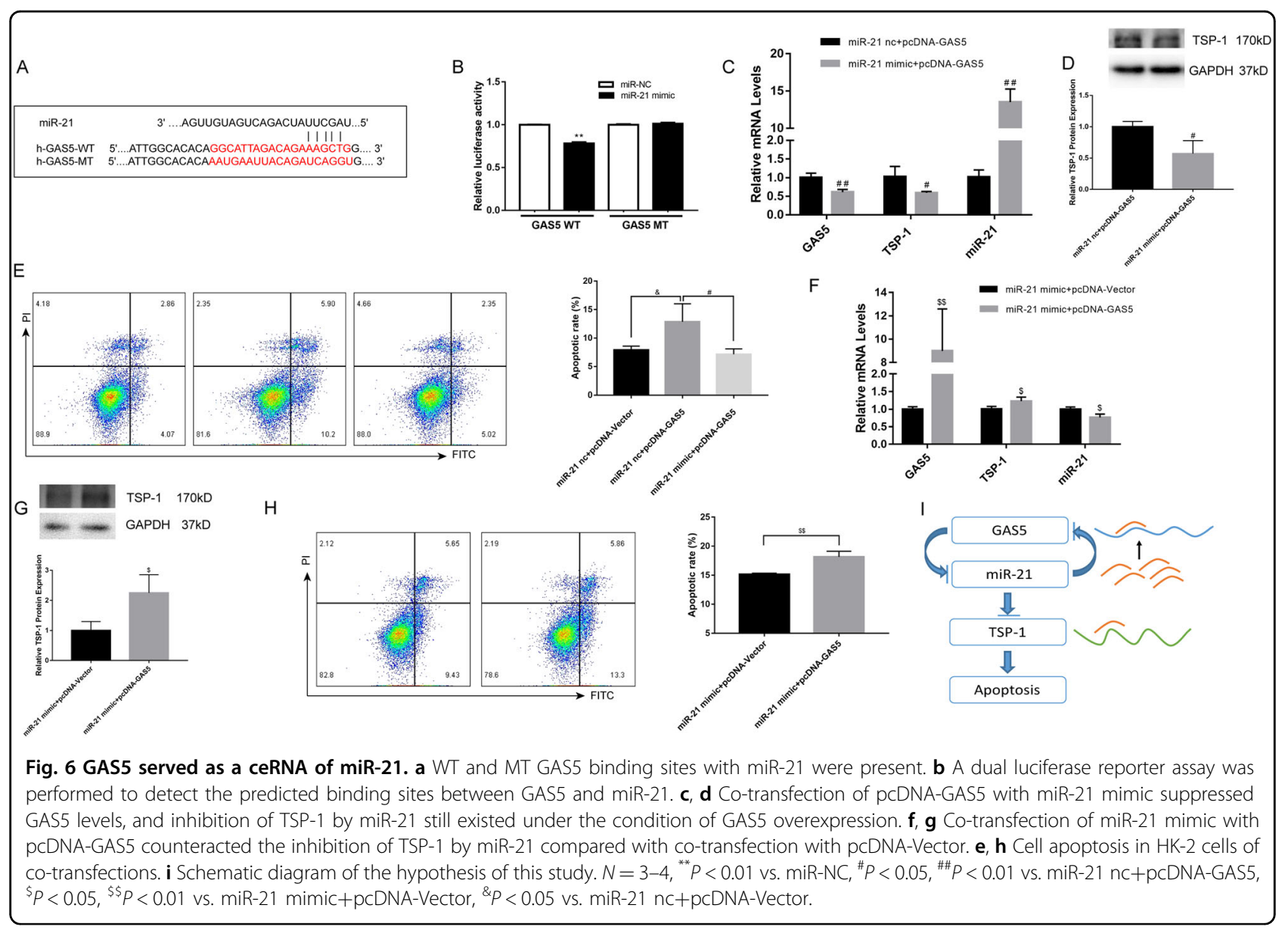

Summarily, we found that GAS5 could indirectly upregulate TSP-1 expression to promote apoptosis by interfering with the function of miR-21 as a ceRNA. Our results revealed novel information regarding the pathogenesis of ischemic AKI.

\section{Materials and methods}

Mouse model of renal ischemia/reperfusion injury and delayed renal ischemic preconditioning

Study protocols were approved by Institutional Animal Care Use Committee of Fudan University. All mice were divided into groups randomly. Briefly, 6- to 8-week-old male C57BL/6 mice were anesthetized with intraperitoneal sodium pentobarbital $(80 \mathrm{mg} / \mathrm{kg})$. Bilateral renal pedicles were clamped for $35 \mathrm{~min}$ in I/R group, followed by various reperfusion intervals. Sham-operated mice underwent anesthesia, laparotomy, and renal pedicle dissection only. Ischemic preconditioning (IPC) and I/R was performed by clamping the bilateral renal pedicles for $15 \mathrm{~min}$ and then for $35 \mathrm{~min} 4$ days later, followed by $24 \mathrm{~h}$ reperfusion. LNA-modified anti-scramble or anti-miR-21 oligonucleotides (Exiqon, USA) $(10 \mathrm{mg} / \mathrm{kg}$ ) were administered intraperitoneally less than $1 \mathrm{~h}$ prior to IPC ${ }^{2}$.
miR-21 Tg+/0 mice were used at 8-10 weeks of age. miR-21 $\mathrm{Tg} 0 / 0$ mice from the same litter were used as controls in the studies. miR-21 $\mathrm{Tg}+/ 0$ mice and $\mathrm{Tg} / 0$ mice were subjected to renal ischemia for $35 \mathrm{~min}$, followed by reperfusion for $24 \mathrm{~h}$.

Kidneys and blood samples were collected at the indicated time. Serum creatinine were measured using a Quantichrom Creatinine Assay Kit (BioAssay Systems, USA).

\section{Histological analysis of renal injury}

Kidney tissues were fixed in $4 \%$ paraformaldehyde, embedded in paraffin, cut into $4-\mu \mathrm{M}$-thick sections and stained with hematoxylin-eosin. Histopathological scoring was performed as previously described ${ }^{11}$.

\section{TUNEL staining}

Paraffin-embedded kidney tissue sections were stained with an In Situ Cell Death Detection Kit (Roche, Switzerland). TUNEL-positive cells were counted at $200 \times$ magnification in 10 fields selected randomly from each slide.

In addition, a TUNEL FITC Apoptosis Detection Kit (Vazyme, China) was used to analyze apoptosis in mice 
kidneys subjected to I/R. Fluorescence microscopy was employed to detect apoptotic cells stained with green fluorescent dye.

\section{In situ hybridization of miR-21 and TSP-1}

In situ hybridization (ISH) was carried out to reveal miR-21 and TSP-1 exposure in Sham- and I/R-induced kidneys. The procedure has been described previously ${ }^{11}$, and the primers are listed in Table 1.

\section{Cell culture, hypoxia/reoxygenation treatment and transfection}

Human renal proximal tubular epithelial (HK-2) cells were obtained from the American Type Culture Collection (ATCC, Manassas, VA) and cultured in Dulbecco's modified Eagle's medium/F12 supplemented with $10 \%$ fetal bovine serum. Cells were grown at $37{ }^{\circ} \mathrm{C}$ with $5 \%$ $\mathrm{CO}_{2}$ and passaged every 3-4 days.

Confluent HK-2 cells were exposed to hypoxia $\left(1 \% \mathrm{O}_{2}\right.$, $5 \% \mathrm{CO}_{2}$, and $94 \% \mathrm{~N}_{2}$ ) for $24 \mathrm{~h}$, followed by $3 \mathrm{~h}$ of reoxygenation (H24R3), or to hypoxia for $6 \mathrm{~h}$, followed by $0.5 \mathrm{~h}$ of reoxygenation (H6R0.5), according to different miR-21 expression levels.

GAS5 and negative control (NC) small interfering RNAs (siRNAs) or miR-21 nc and mimic were transfected into HK-2 cells followed by H24R3 treatment. HK-2 cells transfected with LNA-modified anti-miR-21 and antiscramble (Exiqon, USA) were exposed to H6R0.5 insult. We overexpressed GAS5 by plasmid transfection. All Table 1 Primer sequences for RT-PCR, siRNA sequences
for GAS5 and primer sequences for ISH probes.

\begin{tabular}{ll} 
mouse GAS5 forward & GGATAACAGAGCGAGCGCAAT \\
mouse GAS5 reverse & CCAGCCAAATGAACAAGCATG \\
human GAS5 forward & CTTGCCTGGACCAGCTTAAT \\
human GAS5 reverse & CAAGCCGACTCTCCATACCT \\
mouse TSP-1 forward & GACTCGGGACCCATCTATGA \\
mouse TSP-1 reverse & GGTTATGATTGGCAGCTGATG \\
human TSP-1 forward & GGCAAGGACTGCGTTGGT \\
human TSP-1 reverse & CACTTCACGCCGGCAAAG \\
18S forward & CGGCTACCACATCCAAGGAA \\
18S reverse & CCTGTATTGTATTITCGTCACTACCT \\
human-si-GAS5 sense & CUUGCCUGGACCAGCUUAAUU \\
human-si-GAS5 antisense & UUAAGCUGGUCCAGGCAAGUU \\
miR-21 ISH probe forward & CGCGGGAATTCATTtgtaccaccttgtcgggtag \\
miR-21 ISH probe reverse & GAATTCACTAGTGATgataccaaaatgtcagacagc \\
TSP-1 ISH probe forward & CGCGGGAATTCGATTaaagcctgcaagaaagacgc \\
TSP-1 ISH probe reverse & GAATTCACTAGTGATtgtttgttggccatggcatg \\
\hline
\end{tabular}

transfections were performed using Lipofectamine 3000 (Invitrogen, USA) according to the manufacturer's protocol. In view of cytotoxicity and adverse effects on apoptosis, the dosages of Lipofectamine 3000, plasmids and mimic(nc) were halved when assessing apoptotic rates of co-transfections.

\section{RNA extraction and real-time PCR}

Total RNA was extracted from HK-2 cells and renal tissues using TRIzol reagent (Sigma). For GAS5 and TSP-1, RNA was reverse-transcribed to cDNA using PrimeScript RT Master Mix (Takara, Japan), followed by quantitative analysis using SYBR Premix Ex Taq (Takara, Japan) and an ABI 7500 real-time PCR system. 18S mRNA was used as an internal reference. For miR-21 analysis, RNA was reversetranscribed into cDNA using miRNA-specific primers and a TaqMan MicroRNA Reverse Transcription Kit (Applied Biosystems, USA). Then, cDNA was amplified using TaqMan Universal PCR Master Mix. U6 was used as an endogenous control. Analysis was performed with the $2^{-\Delta \Delta C t}$ method. The primers are listed in Table 1.

\section{Western blot analysis}

Protein obtained using RIPA lysis buffer was separated by $8 \%$ polyacrylamide-SDS gels, transferred to PVDF membranes, blocked with $5 \%$ nonfat milk and then incubated with primary antibodies overnight at $4{ }^{\circ} \mathrm{C}$ followed by secondary antibodies, including horseradish peroxidase-conjugated anti-mouse immunoglobulin G (1:5000; Jackson ImmunoResearch Lab, USA). Primary antibodies included TSP-1 (1:100; Thermo Fisher, USA) and GAPDH (1:2000; Proteintech, USA).

\section{Luciferase reporter assays}

A segment of the $3^{\prime} \mathrm{UTR}$ region of human TSP-1 mRNA, including the predicted miR-21 binding site, was amplified for cloning into the pGL3 basic plasmid downstream of the luciferase reporter gene. Luciferase activities were measured after the transfection.

A fragment from GAS5 containing the predicted miR21 binding site was amplified and then cloned into a psiCHECK2 luciferase vector (Promega, USA), which was named GAS5-wild-type (GAS5-WT). Its counterpart with a mutated miR-21 binding sequence was named GAS5mutated-type (GAS5-MT). Then, GAS5-WT and GAS5MT were co-transfected with miR-21 mimics or miR-21 nc. After transfection for $48 \mathrm{~h}$, the relative luciferase activities were assessed by a Dual-Luciferase Reporter Assay System (Promega, USA).

\section{Flow cytometry}

Apoptosis in HK-2 cells was evaluated by Annexin VFITC/PI double staining (Invitrogen, USA). Cells were harvested and washed with $1 \times$ binding buffer and then 
incubated with Annexin V-FITC for $15 \mathrm{~min}$, followed by PI staining for $5 \mathrm{~min}$ in the dark. Cell apoptosis was analyzed by flow cytometry (Invitrogen, USA).

\section{Statistics}

Statistical analysis was performed using SPSS version 21.0. All data were expressed as mean \pm SD. Comparisons of means between two groups were performed with unpaired Student's two-sided t test. A $P$ value of $<0.05$ was considered statistically significant.

\section{Acknowledgements}

This work was supported by the National Natural Science Foundation of China grants 81770734 (to Dr. Xu) and 81430015 (to Dr. Ding), Science and Technology Commission of Shanghai (14DZ2260200) and Construction Project of Shanghai Renal Disease Clinical Medical Center (2017ZZ01015).

\section{Conflict of interest}

The authors declare that they have no conflict of interest.

\section{Publisher's note}

Springer Nature remains neutral with regard to jurisdictional claims in published maps and institutional affiliations.

The online version of this article (https://doi.org/10.1038/s41420-020-0253-8) contains supplementary material, which is available to authorized users.

Received: 7 November 2019 Revised: 16 January 2020 Accepted: 27 January 2020

Published online: 02 April 2020

\section{References}

1. Bonventre, J. V. \& Yang, L. Cellular pathophysiology of ischemic acute kidney injury. J. Clin. Invest. 121, 4210-4221 (2011).

2. $\mathrm{Xu}, \mathrm{X}$. et al. Delayed ischemic preconditioning contributes to renal protection by upregulation of miR-21. Kidney Int. 82, 1167-1175 (2012).

3. Jia, P. et al. Xenon protects against septic acute kidney injury via miR-21 target signaling pathway. Crit. Care Med. 43, e250-e259 (2015).

4. $\mathrm{Xu}, \mathrm{X}$. et al. Renal protection mediated by hypoxia inducible factor-1alpha depends on proangiogenesis function of miR-21 by targeting thrombospondin 1. Transplantation 101, 1811-1819 (2017).

5. Thakar, C. V. et al. Identification of thrombospondin 1 (TSP-1) as a novel mediator of cell injury in kidney ischemia. J. Clin. Invest. 115, 3451-3459 (2005).

6. Wang, F. et al. Characteristics of long non-coding RNAs in the Brown Norway rat and alterations in the Dahl salt-sensitive rat. Sci. Rep. 4, 7146 (2014).

7. YN, W. et al. Long noncoding RNA-GAS5: a novel regulator of hypertensioninduced vascular remodeling. Hypertension 68, 736-748 (2016).
8. Chang, Y. N. et al. Hypoxia-regulated IncRNAs in cancer. Gene 575, 1-8 (2016).

9. Zhou, P., Chen, Z., Zou, Y. \& Wan, X. Roles of non-coding RNAs in acute kidney injury. Kidney Blood Press Res. 41, 757-769 (2016).

10. Yu, T. M. et al. RANTES mediates kidney ischemia reperfusion injury through a possible role of HIF-1alpha and LncRNA PRINS. Sci. Rep. 6, 18424 (2016).

11. Geng, X. et al. The effect of long noncoding RNA GAS5 on apoptosis in renal ischemia/reperfusion injury. Nephrology 24, 405-413 (2019).

12. Zhang, Z. et al. Negative regulation of IncRNA GAS5 by miR-21. Cell Death Differ. 20, 1558-1568 (2013).

13. Ma, C. et al. The growth arrest-specific transcript 5 (GAS5): a pivotal tumor suppressor long noncoding RNA in human cancers. Tumour Biol. 37, 1437-1444 (2016).

14. Pickard, M. R. \& Williams, G. T. Molecular and cellular mechanisms of action of tumour suppressor GAS5 LncRNA. Genes 6, 484-499 (2015).

15. Lin, J. et al. The long noncoding RNA landscape in hypoxic and inflammatory renal epithelial injury. Am. J. Physiol. Ren. Physiol. 309, F901-F913 (2015).

16. Tian, $X$. et al. LINC00520 targeting miR-27b-3p regulates OSMR expression level to promote acute kidney injury development through the PI3K/AKT signaling pathway. J. Cell Physiol. 234, 14221-14233 (2019).

17. Liu, $X$. et al. Long non-coding RNA GAS5 acts as a molecular sponge to regulate miR-23a in gastric cancer. Minerva Med. (2016).

18. Zhang, X. F., Ye, Y. \& Zhao, S. J. LncRNA Gas5 acts as a ceRNA to regulate PTEN expression by sponging miR-222-3p in papillary thyroid carcinoma. Oncotarget 9, 3519-3530 (2018).

19. Chen, F., Zhang, L., Wang, E., Zhang, C. \& Li, X. LncRNA GAS5 regulates ischemic stroke as a competing endogenous RNA for miR-137 to regulate the Notch1 signaling pathway. Biochem. Biophys. Res. Commun. 496, 184-190 (2018).

20. Godwin, J. G. et al. Identification of a microRNA signature of renal ischemia reperfusion injury. Proc. Natl Acad. Sci. USA 107, 14339-14344 (2010).

21. Ren, G. L., Zhu, J., Li, J. \& Meng, X. M. Noncoding RNAs in acute kidney injury. J. Cell Physiol. 234, 2266-2276 (2019).

22. Jia, P. et al. miR-21 contributes to xenon-conferred amelioration of renal ischemia-reperfusion injury in mice. Anesthesiology 119, 621-630 (2013).

23. Pan, T. et al. Delayed remote ischemic preconditioning confersrenoprotection against septic acute kidney injury via exosomal miR-21. Theranostics 9 , 405-423 (2019).

24. Du, J. et al. MicroRNA-21 and risk of severe acute kidney injury and poor outcomes after adult cardiac surgery. PLoS One 8, e63390 (2013).

25. $\mathrm{Xu}, \mathrm{X}$. et al. miR-21 in ischemia/reperfusion injury: a double-edged sword? Physiol. Genomics 46, 789-797 (2014).

26. Bige, N. et al. Thrombospondin-1 plays a profibrotic and pro-inflammatory role during ureteric obstruction. Kidney Int. 81, 1226-1238 (2012).

27. Bhattacharyya, S., Marinic, T. E., Krukovets, I., Hoppe, G. \& Stenina, O. I. Cell typespecific post-transcriptional regulation of production of the potent antiangiogenic and proatherogenic protein thrombospondin-1 by high glucose. J. Biol. Chem. 283, 5699-5707 (2008).

28. Rogers, N. M., Thomson, A. W. \& Isenberg, J. S. Activation of parenchymal CD47 promotes renal ischemia-reperfusion injury. J. Am. Soc. Nephrol. 23, 1538-1550 (2012).

29. Rogers, N. M., Zhang, Z. J., Wang, J. J., Thomson, A. W. \& Isenberg, J. S. CD47 regulates renal tubular epithelial cell self-renewal and proliferation following renal ischemia reperfusion. Kidney Int. 90, 334-347 (2016). 